\title{
Detection of Influenza Viruses Attached to an Optical Disk
}

\author{
Takayuki Shima ${ }^{1}$, Makoto Fujimaki ${ }^{1}$, Akihiro Yoshida ${ }^{2}$, Subash C. B. Gopinath ${ }^{1}$, Masashi Kuwahara ${ }^{1}$, \\ Yoshimichi Ohki ${ }^{2}$, Koichi Awazu ${ }^{1}$ \\ ${ }^{1}$ Electronics and Photonics Research Institute (ESPRIT), National Institute of Advanced Industrial Science and Technology (AIST), \\ Tsukuba, Japan; ${ }^{2}$ Department of Electrical Engineering and Bioscience, Waseda University, Tokyo, Japan. \\ Email: t-shima@aist.go.jp
}

Received January $15^{\text {th }}, 2013$; revised March $1^{\text {st }}, 2013$; accepted April $4^{\text {th }}, 2013$

Copyright (C) 2013 Takayuki Shima et al. This is an open access article distributed under the Creative Commons Attribution License, which permits unrestricted use, distribution, and reproduction in any medium, provided the original work is properly cited.

\begin{abstract}
Detection of polystyrene beads with a diameter of $100 \mathrm{~nm}$ was conducted using an optical disk drive tester equipped with a laser of $405 \mathrm{~nm}$ wavelength and an objective lens with a numerical aperture of 0.65 . The polystyrene beads were used to mimic the influenza virus. A grooved disk with a $(\mathrm{ZnS})_{85}\left(\mathrm{SiO}_{2}\right)_{15} / \mathrm{SiO}_{2}$ layered surface structure was used for the detection. The detection of influenza viruses (A/Udorn/307/1972) with Au nanoparticles was also demonstrated using the optical disk drive tester. In this case, a grooved disk with an indium tin oxide (ITO) film was used. The ITO film functioned both to tune the reflectance of the disk and as an electrically conductive layer for scanning electron microscopy. In both cases, the target substances were successfully recognized in a single scan with a high scanning speed of $4.9 \mathrm{~m} / \mathrm{s}$. The results indicate that this optical disk system can be used to detect $100 \mathrm{~nm}$ scale substances like influenza viruses, which are smaller than the diffraction limit of the system.
\end{abstract}

Keywords: Influenza Virus; Polystyrene Bead; Optical Disk; Laser; Reflectance; Sensor; Safety and Security

\section{Introduction}

The diagnosis of influenza is usually made after infection. When viruses are detected in some individuals, the number of influenza patients may already be increasing, including those during the latent stage of infection. Such epidemics occasionally and severely threaten the safety and security of life. Quick detection of any signs of influenza is thus desired, preferably when the virus is airborne before people become infected.

In order to detect viruses in the air, such as those existing in the droplet of a sneeze, we focused on using optical disk technology originally used for playing music and videos. Signals recorded on an optical disk are pits of various lengths. The shortest pit length is $150 \mathrm{~nm}$ in the 25 GB Blu-ray Disc (BD). This length is close to the diameter of the influenza virus, expected to be about $100 \mathrm{~nm}$. Therefore, we expect that optical disk technology can "read" signals from such a virus [1].

Much research work has been published that use optical disk technology in order to detect biological substances. Varma et al. prepared Au radial spokes on the disk, and measured optical interference in order to detect an antigen-antibody reaction of immunoglobulin G [2]. Hemmi et al. made a disk-shaped microfluidic device in order to mix solutions by the centrifugal force generated during disk rotation. Here, a surface plasmon resonance sensor was included to detect an antigen-antibody reaction of immunoglobulin A [3]. Gopinath et al. optimized the stacking of $\mathrm{Au}, \mathrm{AgInSbTe}$ and $\mathrm{ZnS}-\mathrm{SiO}_{2}$ films on a conventional optical disk substrate, and measured reflected light intensity at a high disk-rotation speed $(6 \mathrm{~m} / \mathrm{s})$ for detecting the antigen-antibody reaction of the influenza A virus [4]. These previous works are all similar from the viewpoint of detecting the assembly of target substances.

This study is intended to detect the target "one by one" using optical disk technology without major modifications to the system. In the present report, the detection of polystyrene beads with a diameter of $100 \mathrm{~nm}$ and influenza viruses is demonstrated. The results are compared with actual images obtained by a scanning electron microscope (SEM). Although the proposed method is simple, the number of targets becomes countable, and one may return to the position of the detected target for further analyses [5].

\section{Experimental Details}

We used a polycarbonate disk substrate with a diameter 
of $12 \mathrm{~cm}$ and a thickness of $0.6 \mathrm{~mm}$. The disk has grooves on the surface for a readout laser to trace from the rear side. The width, period and depth of the grooves were $340 \mathrm{~nm}, 680 \mathrm{~nm}$ and $39 \mathrm{~nm}$, respectively. Transparent films were deposited on the grooves by radio frequency (RF) magnetron sputtering at room temperature. For Disk A, a $(\mathrm{ZnS})_{85}\left(\mathrm{SiO}_{2}\right)_{15}$ film was deposited on the grooves and the film surface was coated with a $\mathrm{SiO}_{2}$ thin film. $(\mathrm{ZnS})_{85}\left(\mathrm{SiO}_{2}\right)_{15}$ was chosen since it is commonly used as a dielectric material in the optical disk [6]. A $\mathrm{SiO}_{2}$ film was for introducing surface modification using silane coupling agents for selective adsorption of specific targets, but was not used in this way in the present experiment. The film thicknesses were adjusted to show nearly a maximum reflectance at a laser wavelength of $405 \mathrm{~nm}$. Disk A was used for the detection of polystyrene beads. For Disk B, an indium tin oxide (ITO) film was deposited on the grooves. Since ITO is electrically conductive [7], charge effects can be reduced during SEM observation of the target. Disk B was used for the detection of influenza viruses. The experimental conditions are summarized in Table 1.

We first created marks on the disks by high-power laser irradiation. The marks that can be recognized by the optical disk system and SEM observation were necessary to specify the position on the disks. For example, various length marks, centered at either $2 \mu \mathrm{m}$ or $100 \mu \mathrm{m}$, were recorded at $12 \mathrm{~mW}$ with the disk rotation speed of 1.1 $\mathrm{m} / \mathrm{s}$ for Disk A, and at $8.5 \mathrm{~mW}$ with $2.2 \mathrm{~m} / \mathrm{s}$ for Disk B. The mark recording mechanism is not well studied, but is presumably due to volume expansion and/or desorption of disk materials during a local increase in temperature by laser irradiation $[8,9]$. Thus the reflectance of the mark may rise (or fall) with no uniformity; however, it is still sufficient in this study as far as its position on the disk can be identified.

A solution containing polystyrene beads was obtained from Polysciences Inc. The mean diameter of the beads was $100 \mathrm{~nm}$. The concentration was $4.55 \times 10^{13}$ particles/ $\mathrm{ml}$, and the solution was diluted $10^{4}$ times by de-ionized water. A droplet of the diluted solution was placed on Disk A and the disk was dried at room temperature before the evaluation.

For the virus detection experiments, an influenza virus (A/Udorn/307/1972) was used. A solution containing the virus $(5 \mu \mathrm{l})$ was mixed with a solution containing antihaemagglutinin antibody-attached $10 \mathrm{~nm}$ Au nanoparticles $(50 \mu \mathrm{l})$ and phosphate buffered saline (PBS, $450 \mu \mathrm{l})$. After waiting $10 \mathrm{~min}$ for the antigen-antibody reaction, PBS was further added in order to make a $2 \mathrm{ml}$ solution. The virus concentration was $7.4 \times 10^{9} \mathrm{pfu} / \mathrm{ml}$ (plaque forming unit per milliliter) in the beginning, and thus diluted to be $1.9 \times 10^{7} \mathrm{pfu} / \mathrm{ml}$ in the final virus solution. $\mathrm{Au}$ nanoparticles were used to identify viruses from other
Table 1. Summary of the experiments in this study.

\begin{tabular}{|c|c|c|}
\hline & Disk A & Disk B \\
\hline Film(s) & $\begin{array}{l}(\mathrm{ZnS})_{85}\left(\mathrm{SiO}_{2}\right)_{15} \\
120 \mathrm{~nm} / \mathrm{SiO}_{2} 5 \mathrm{~nm}\end{array}$ & ITO $140 \mathrm{~nm}$ \\
\hline Target & $\begin{array}{l}\text { Polystyrene bead } \\
\text { (diameter: } 100 \mathrm{~nm} \text { ) }\end{array}$ & $\begin{array}{l}\text { Influenza virus } \\
\text { (A/Udorn/307/1972) }\end{array}$ \\
\hline Additive & None & $\begin{array}{l}\text { Antibody-attached } \mathrm{Au} \\
\text { nano-particle (particle } \\
\text { diameter: } 10 \mathrm{~nm})\end{array}$ \\
\hline Density & $4.55 \times 10^{9} / \mathrm{ml}$ & $1.9 \times 10^{7} \mathrm{pfu} / \mathrm{ml}$ \\
\hline Target supply & Solution dropped & Solution sprayed \\
\hline
\end{tabular}

substances included in the solutions. A nebulizer (Voyage, Air Liquide Medical Systems) was used to spray the virus solution onto Disk B. The spraying experiment was done inside a draft chamber, and it took about $30 \mathrm{~min}$ to spray most of the prepared virus solution. After drying the disk at room temperature, the disk was rinsed with de-ionized water for removing any precipitated salts, and then dried again.

An optical disk drive tester (DDU-1000, Pulstec Industrial) with a laser wavelength $(\lambda)$ of $405 \mathrm{~nm}$ and a numerical aperture (NA) of 0.65 was used for the detection of the polystyrene beads and the viruses. The optics were designed for a High-Definition Digital Versatile Disc (HD DVD) system. The disk rotation speed for readout was $4.9 \mathrm{~m} / \mathrm{s}$, which is similar to it of BD. The laser power for readout was fixed at $1.0 \mathrm{~mW}$. An oscilloscope (Wavepro 7300, LeCroy) was used to collect the reflected light intensity data obtained by the optical disk drive tester.

\section{Results and Discussion}

Curves (a) and (b) in Figure 1 show the calculated reflectance using the Fresnel equations simulating materials with refractive indices of 1.63 and 1.45 attached to Disk A and Disk B, respectively. In the calculations, the

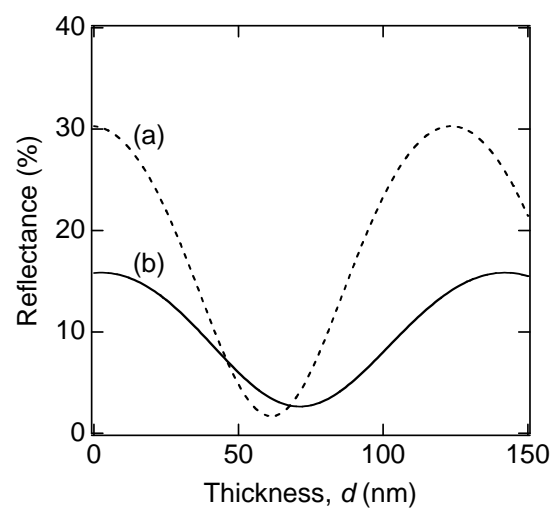

Figure 1. Calculated results for films with a refractive index $n$ of (a) 1.63 and (b) 1.45 attached to Disk A and Disk B, respectively. 
materials are assumed as films of thickness $d$. As mentioned in the previous section, the thicknesses of the $(\mathrm{ZnS})_{85}\left(\mathrm{SiO}_{2}\right)_{15}$ and the $\mathrm{SiO}_{2}$ films for Disk $\mathrm{A}$ and that of ITO for Disk B were adjusted in order to obtain nearly the highest reflectance when nothing is attached on the disk surface, i.e., $d=0$. As shown in Figure 1, the reflectance changes sinusoidally with a change in $d$. The refractive index of polystyrene is 1.63 at a wavelength of $405 \mathrm{~nm}$ [10]. The exact refractive index of the virus is not known, but it was assumed as 1.45 , similar to the one of a protein [11]. The diameter of the bead is $100 \mathrm{~nm}$, a similar size as that expected for the virus. According to these values and the results shown in Figure 1, the reflectance should decrease when the targets are attached to the disk surface for detection.

Figure 2 shows a SEM image of the polystyrene beads attached to Disk A. White spots that correspond to the beads can be seen on the grooved structure of the disk. In certain areas, the beads are rather aggregated (not shown in Figure 2), and we here selected an area where the beads are dispersed near the mark formed. The laser light of the tester was irradiated along the scanning direction shown in the figure. There were three beads labelled A1, $\mathrm{A} 2$ and $\mathrm{A} 3$, and the mark labelled Mark in this order on the laser-scanned groove. The distances between $\mathrm{A} 1$ and $\mathrm{A} 2$, and, $\mathrm{A} 2$ and $\mathrm{A} 3$ are $1.8 \mu \mathrm{m}$ and $3.0 \mu \mathrm{m}$, respectively, according to the length scale of the SEM.

Figure 3 shows the reflected light intensity when the laser light scanned the groove in Figure 2. The data is shown in units of voltage since an oscilloscope was used. The very large signals seen at the right hand side of the figure is from Mark and some beads in its vicinity, and the intensity of Mark fluctuated due to its lack of uniformity. Three dips of A1', A2' and A3' were observed in the scan, and the distances between them were calculated using the disk rotation speed of $4.9 \mathrm{~m} / \mathrm{s}$. The distances between $\mathrm{A} 1$ ' and $\mathrm{A} 2$ ', and, $\mathrm{A} 2$ ' and $\mathrm{A} 3^{\prime}$ are 1.8 $\mu \mathrm{m}$ and $3.3 \mu \mathrm{m}$, respectively. They are similar to the distance data obtained in Figure 2, and one can consider that the signals of A1', A2', and A3' are due to the parti- cles A1, A2, and, A3, respectively. The results in Figures 2 and 3 demonstrate that the $100 \mathrm{~nm}$ beads can be detected using the optical disk technology. Although the $100 \mathrm{~nm}$ length is less than the resolution limit of the optics $(\lambda / 4 \mathrm{NA}=156 \mathrm{~nm})$ used [12], the beads in Figure 2 were possible to detect because they were isolated. The signal-to-noise quality shown in Figure $\mathbf{3}$ may not be enough; however, we can easily improve it by applying multiple scanning.

The peak width $w$ of A1' was about $750 \mathrm{~nm}$, much larger than the bead diameter of $100 \mathrm{~nm}$. This discrepancy may be understood by considering the laser spot diameter. The laser spot size is expected to be about 620 $\mathrm{nm}(=\lambda / \mathrm{NA})$ in the optics used. The bead will be detected at the forefront of the laser spot that reaches it, and detection continues until the back end of the laser spot departs from it. Thus, the laser spot size should be added to the beam diameter for the analysis of the peak width, and $720 \mathrm{~nm}$ is fairly close to what is observed.

Figure 4 shows a SEM image when the mixed virus/ Au nanoparticle solution is dropped on a flat Si substrate and dried. This experiment was done in order to understand how the dried solution would be observed in a SEM image. The small white spots are Au particles. Two patterns were observed on the dispersion. One pattern was isolated (circle in the figure), and the other was aggregated on a convex spot (dashed-line square in the figure). The convex spot was considered to be the influenza virus since the size was about $100 \mathrm{~nm}$ and Au aggregation is expected when the antigen-antibody reaction occurs at the surface of the virus. Accordingly, one can identify whether the attached substances are viruses or not from the Au aggregation.

Figure 5 shows a SEM image of the influenza virus with the Au nanoparticles on Disk B when sprayed. All images in Figure 5 were less influenced by charging effects as compared to Figure 2. At the low magnification of Figure 5(a), very faint dark spots of B1 and B2 are noticeable. The higher magnification images in Figures 5(b) and (c) indicate the aggregation of $\mathrm{Au}$ nano-

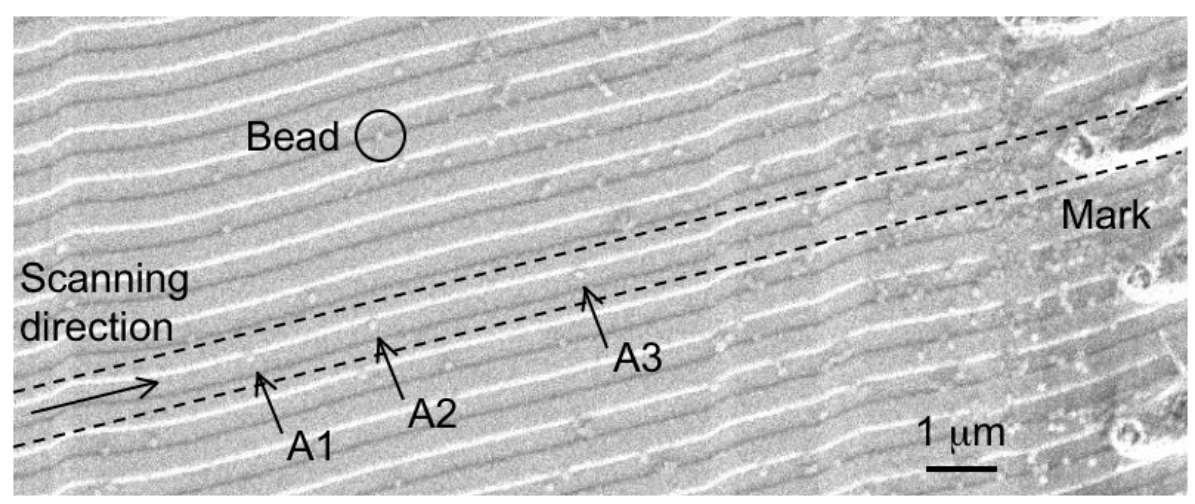

Figure 2. SEM image of the polystyrene beads (diameter: $100 \mathrm{~nm}$ ) dispersed on Disk A. 


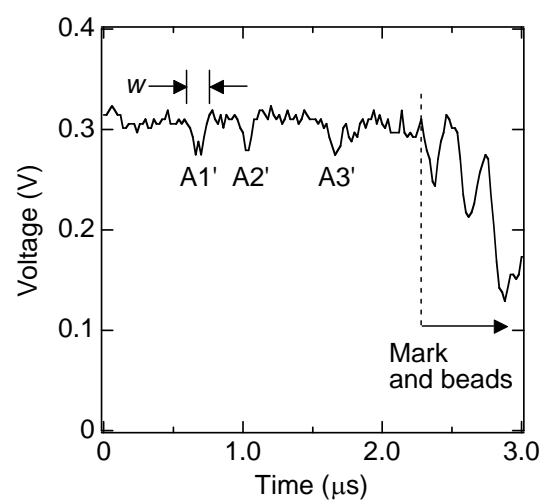

Figure 3. Reflected light intensity as recorded by an oscilloscope when the laser scanned the groove in Figure 2.

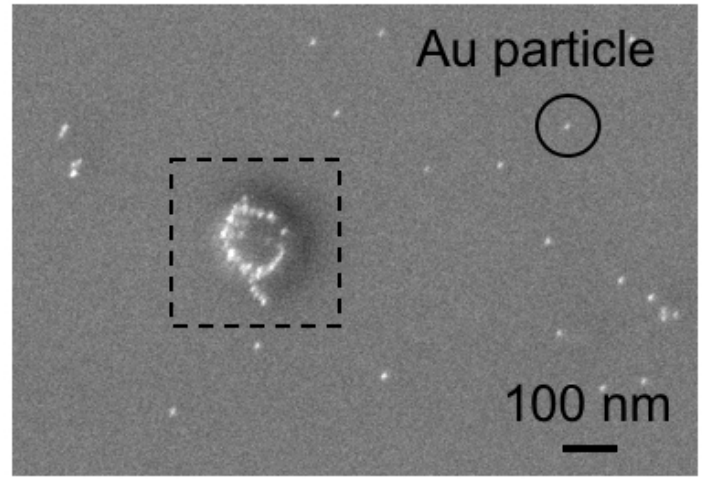

Figure 4. SEM image of a dried solution of influenza virus anti-body/Au nanoparticle on a Si substrate.
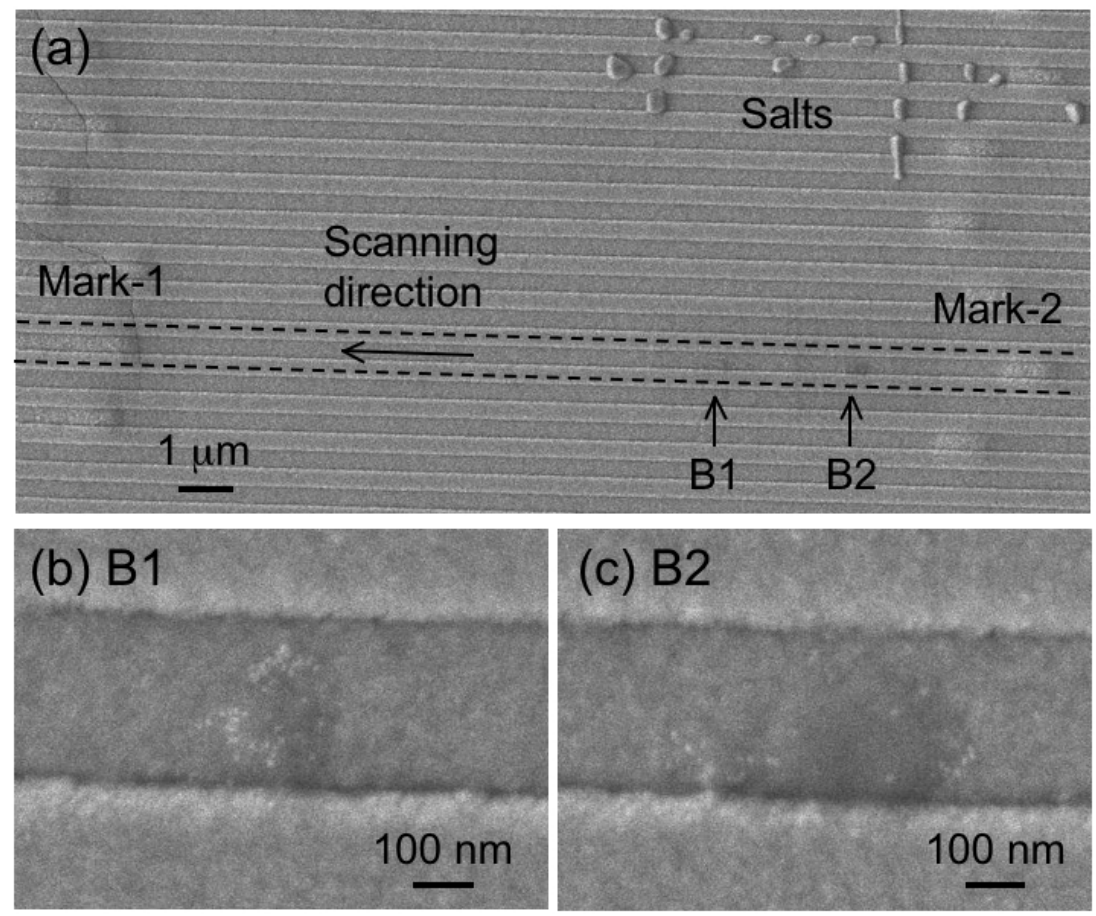

Figure 5. (a) SEM image of the influenza viruses dispersed on Disk B, (b) and (c) are expanded images of B1 and B2 in Figure 5(a), respectively.

particles at B1 and B2. Aggregated Au nanoparticles are considered to mark viruses. The number of viruses seen in Figure 5(c) could be more than one. In addition to viruses, other substances were observed in the image. We believe that these foreign substances are salts included in the solution that partly remained after the rinsing. Two marks (Mark-1 and Mark-2) are observed in Figure 5(a), and the distances between the edge of Mark-1 and B1, B1 and B2, and B2 and Mark-2 are $10.2 \mu \mathrm{m}, 2.5 \mu \mathrm{m}$ and $2.9 \mu \mathrm{m}$, respectively from the SEM image.

Figure 6 shows the reflected light intensity when the laser light scanned the groove in Figure 5, where the laser light scanned Mark-2, B2, B1 and Mark-1 along the groove in this order. The signals due to the marks fluctu- ated for the same reason explained in Figure 3. In Figure 6, two dips of $\mathrm{B} 1$ ' and $\mathrm{B} 2$ ' were observed, and the distances between the edge of Mark-1 and B1', B1' and B2', and B2' and Mark-2 are $10.6 \mu \mathrm{m}, 2.4 \mu \mathrm{m}$ and 3.3 $\mu \mathrm{m}$, respectively. These distances in Figures $\mathbf{5}$ and $\mathbf{6}$ are similar, and thus one can consider that the signals of $\mathrm{B} 1^{\prime}$ and B2' are due to the viruses B1 and B2, respectively.

In Figure 6, it is a question as to whether the viruses or Au nanoparticles are detected. The length of the virus spot B2 along the groove in Figure 5(c) was longer than that of the virus spot B1 in Figure 5(b). A similar relation can be found in the signal width of B1' and B2' in Figure 6. The intensity reduction at $\mathrm{B} 1^{\prime}(44 \mathrm{mV})$ and B2' $(53 \mathrm{mV})$ in Figure 6 was fairly similar, although 


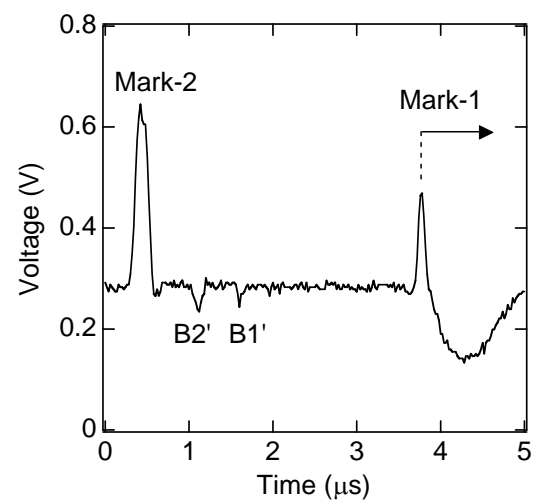

Figure 6. Reflected light intensity recorded by an oscilloscope when the laser scanned the groove in Figure 5(a).

there were apparently more $\mathrm{Au}$ nanoparticles aggregated at B1 than B2 in Figure 5. It is thus more likely that the virus itself was detected in Figure 6. Nonetheless, further quantitative experiments may be necessary in order to give a clear conclusion. As an additional experiment, we have dropped a solution of Au nanoparticles without viruses on Disk B. The particles could not be optically detected although they were dispersed on the scanned grooves, probably due the small particle size $(10 \mathrm{~nm})$.

The results in Figures 5 and $\mathbf{6}$ demonstrated that influenza viruses could be detected "one by one" using the optical disk technology described in the present paper. Here, "one" may refer to one virus or one aggregate of viruses that is expelled as a unit from the nebulizer. To the best of our knowledge, whether viruses are isolated or aggregated as they float in air is not well understood. We believe that the detection of even one virus is possible from the results in Figures $\mathbf{2}$ and $\mathbf{3}$ since $100 \mathrm{~nm}$ diameter substances were well measured.

We note that the salts observed in the upper part of Figure 5(a) could also be detected by the optical disk drive tester. In future work, one needs to understand how to distinguish viruses from other substances when using the optical disk technology as a virus detector. We believe that there are at least three possible ways to distinguish viruses from other substances. First, a more quantitative analysis on the reflectance change, such as the intensity and the width of the signals, should be performed. If the refractive index and shape of a target is known and optical reflectance taking its three-dimensional structure into account is feasible to be calculated, it becomes easier to differentiate the target signal from that of other materials. Second, additional structures on the disk should be fabricated for selective adsorption of the target, e.g. introduction of antibodies using surface modification. In this case, it is important that such a structure functions in the environment that the optical disk sensor is used, such as dry conditions. Third, further analyses of the target after the first detection should be performed. Raman scattering may be a powerful tool to distinguish the various detected target materials [13]. Although much work remains in order to realize the practical use of the proposed sensor, this method has the potential of detecting $100 \mathrm{~nm}$ scale substances at high speed, owing partly to present optical disk information technology.

\section{Conclusion}

We have examined the detection of $100 \mathrm{~nm}$ polystyrene beads and influenza viruses using an optical disk drive tester. We have successfully detected these particles using this method. The results agreed well with images taken by a SEM. This result indicates that the optical disk technology can be used for the detection system of airborne viruses. In addition, this system can be used to recognize any particle-shape materials with a known complex refractive index and size.

\section{Acknowledgements}

The authors would like to thank Dr. K. Shimizu of Nihon University for supplying us the influenza virus. The authors also thank H. Saito of ESPRIT, AIST for his technical assistance in the disk sample preparation. This work was partially supported by JSPS KAKENHI Grant Number 24656068 .

\section{REFERENCES}

[1] T. Noda, H. Sagara, A. Yen, A. Takada, H. Kida, R. H. Cheng and Y. Kawaoka, "Architecture of Ribonucleoprotein Complexes in Influenza a Virus Particles," Nature, Vol. 439, No. 7075, 2006, pp. 490-492. doi: $10.1038 /$ nature 04378

[2] M. M. Varma, D. D. Nolte, H. D. Inerowicz and F. E. Regnier, "Spinning-Disk Self-Referencing Interferometry of Antigen-Antibody Recognition," Optics Letters, Vol. 29, No. 9, 2004, pp. 950-952. doi:10.1364/OL.29.000950

[3] A. Hemmi, T. Usui, A. Moto, T. Tobita, N. Soh, K. Nakano, H. Zeng, K. Uchiyama, T. Imato and H. Nakajima, "A Surface Plasmon Resonance Sensor on a Compact Disk-Type Microfluidic Device," Journal of Separation Science, Vol. 34, No. 20, 2011, pp. 2913-2919. doi: $10.1002 /$ jssc. 201100446

[4] S. C. B. Gopinath, K. Awazu, P. Fons, J. Tominaga and P. K. R. Kumar, "A Sensitive Multilayered Structure Suitable for Biosensing on the BioDVD Platform," Analytical Chemistry, Vol. 81, No. 12, 2009, pp. 4963-4970. doi: $10.1021 / \mathrm{ac} 802757 \mathrm{z}$

[5] M. Kuwahara, P. Fons, J. Tominaga, K. Honma, A. Egawa, T. Miyatani, K. Nakajima, H. Abe and H. Tokumoto, "Development of a Geometrical Evaluation Apparatus for Ultrahigh 100 GB Optical Disk Masters," Review of Scientific Instruments, Vol. 76, No. 8, 2005, Article ID: 083706. doi:10.1063/1.1994921 
[6] M. Takao, K. Nagata, K. Kimura and T. Kurumizawa, “Optical Recording Material," Japan Patent No. 1788207, 1993.

[7] D. B. Fraser and H. D. Cook, "Highly Conductive, Transparent Films of Sputtered $\operatorname{In}_{2-\mathrm{x}} \mathrm{Sn}_{\mathrm{x}} \mathrm{O}_{3-\mathrm{y}}$," Journal of the Electrochemical Society, Vol. 119, No. 10, 1972, pp. 1368-1374. doi:10.1149/1.2403999

[8] H. Miura, N. Toyoshima, Y. Hayashi, S. Sangu, N. Iwata

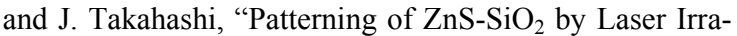
diation and Wet Etching," Japanese Journal of Applied Physics, Vol. 45, No. 2B, 2006, pp. 1410-1413. doi:10.1143/JJAP.45.1410

[9] W. F. Wu and B. S. Chiou, "Effect of Annealing on Electrical and Optical Properties of RF Magnetron Sputtered Indium Tin Oxide Films," Applied Surface Science, Vol. 68, No. 4, 1993, pp. 497-504. doi:10.1016/0169-4332(93)90233-2

[10] S. N. Kasarova, N. G. Sultanova, C. D. Ivanov and I. D.
Nikolov, "Analysis of the Dispersion of Optical Plastic Materials," Optical Materials, Vol. 29, Vol. 11, 2007, pp. 1481-1490. doi:10.1016/j.optmat.2006.07.010

[11] S. Busse, V. Scheumann, B. Menges and S. Mittler, "Sensitivity Studies for Specific Binding Reactions Using the Biotin/Streptavidin System by Evanescent Optical Methods," Biosensors and Bioelectronics, Vol. 17, No. 8, 2002, pp. 704-710. doi:10.1016/S0956-5663(02)00027-1

[12] T. Kikukawa, T. Kato, H. Shingai and H. Utsunomiya, "High-Density Read-Only Memory Disc with Super Resolution Reflective Layer," Japanese Journal of Applied Physics, Vol. 40, No. 3B, 2001, pp. 1624-1628. doi:10.1143/JJAP.40.1624

[13] M. Kuwahara, T. Shima, P. Fons and J. Tominaga, "InSitu Raman Scattering Spectroscopy for a Super Resolution Optical Disk During Readout," Applied Physics Express, Vol. 2, No. 8, 2009, Article ID: 082402. doi:10.1143/APEX.2.082402 